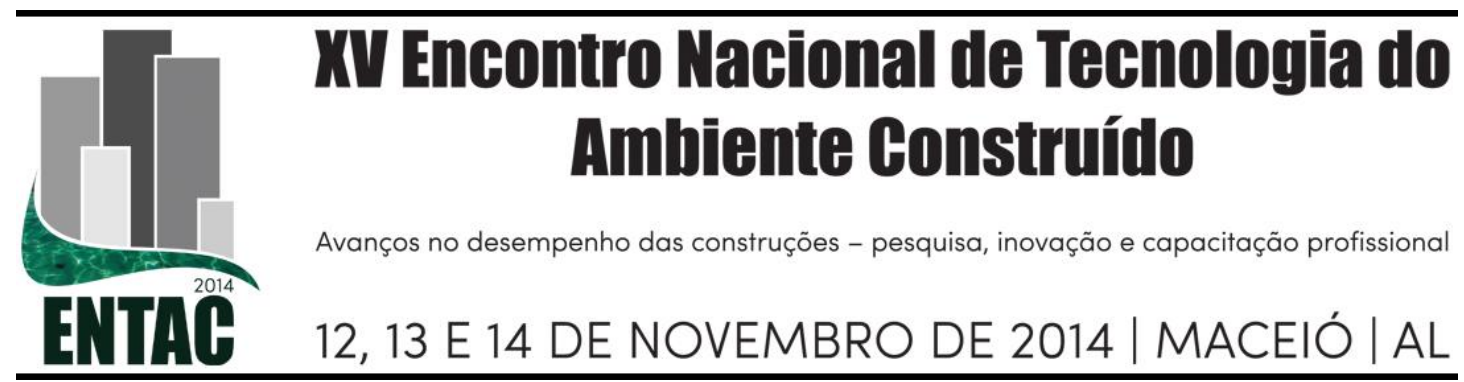

\title{
PROPOSTA DE AJUSTE NA ETIQUETAGEM DO NÍVEL DE EFICIÊNCIA ENERGÉTICA DE EDIFICAÇÕES RESIDENCIAIS
}

\author{
WESTPHAL, Fernando Simon
}

(1) Universidade Federal de Santa Catarina, (48) 3721-9550, e-mail: fernando.sw@ufsc.br

\begin{abstract}
RESUMO
O Regulamento Técnico da Qualidade para o Nível de Eficiência Energética de Edificações Residenciais (RTQ-R), publicado pelo INMETRO em 2012, contém dois procedimentos de avaliação do desempenho de edificações: um método prescritivo e um método por simulação. $\mathrm{O}$ método prescritivo consiste na aplicação de equações para o cálculo de graus-hora de desconforto e consumo de energia para resfriamento e aquecimento da unidade habitacional em análise. Por meio delas, indicadores de desempenho da edificação são relacionados com parâmetros que descrevem o projeto da edificação. Porém, a lista de variáveis não leva em conta o tipo de vidro ou o fator solar de fechamentos envidraçados. O objetivo deste trabalho é propor novas equações de cálculo para o RTQ-R, incluindo o fator solar como o parâmetro que descreve a influência do vidro no nível de eficiência da edificação. Uma série de simulações computacionais paramétricas foi realizada com o programa EnergyPlus e arquivos climáticos de dados horários de oito cidades brasileiras cobrindo todas as zonas bioclimáticas definidas na NBR 15220. Novas equações para descrever o desempenho de edificações residenciais foram obtidas por análise de regressão reproduzindo a mesma metodologia aplicada pelo CB3E (Centro Brasileiro de Eficiência Energética em Edificações), quando da obtenção das equações para desenvolvimento da versão atual do RTQ-R. Três diferentes especificações de vidro foram utilizadas para ajustar o lote de simulações adotado naquela metodologia. Como resultados, as novas equações foram geradas e os coeficientes de determinação $\left(\mathrm{R}^{2}\right)$ obtidos foram iguais ou melhores do que o modelo atual do RTQ-R. O melhor modelo foi alcançado para a Zona Bioclimática 2 , onde o $\mathrm{R}^{2}$ foi de 0,91 nesta proposta, contra 0,82 do modelo atual. O trabalho contribui para aprimorar o modelo de avaliação do nível de eficiência energética de edificações, considerando os benefícios de vidros de controle solar em residências.
\end{abstract}

Palavras-chave: etiquetagem, edificações residenciais, vidros.

\begin{abstract}
The National Regulations for Energy Efficiency of Residential Buildings (RTQ -R ), published in 2012 by INMETRO contains two procedures for evaluating the performance of buildings: a prescriptive method and a method by simulation. The prescriptive method consists of applying equations for calculating degree- hours of discomfort and energy consumption for cooling and heating of the housing unit under analysis. Through the equations, building performance indicators are related to parameters that describe the building design. However, the list of parameters does not take into account the type of glass or the solar factor of the glazing system installed in the façade. The aim of this work is to propose new equations for the $R T Q-R$, including the solar factor as the parameter that describes the influence of the glass in the energy efficiency level of the building. A set of parametric simulations were carried out with EnergyPlus software and weather files of eight Brazilian cities covering all bioclimatic zones defined in NBR 15220. New equations to describe the performance of residential buildings were obtained by regression analysis replicating the same methodology applied by CB3E (Brazilian Center for Energy Efficiency in Buildings), when obtaining the equations for the current development version of RTQ-R. Three different specifications of glass were adopted to adjust the batch simulations performed in that methodology. As a result, the new equations were generated and the coefficients of determination $\left(R^{2}\right)$ obtained were equal to or better than the current model of the RTQ-R. The best model was achieved for Bioclimatic Zone 2, where $R^{2}$ was 0.91 in this proposal, against 0.82 for the current model. This work
\end{abstract}


contributes to enhance the model to assess the level of energy efficiency of buildings, considering the benefits of solar control glazing in residential buildings.

Keywords: labeling, residential buildings, glazing.

\section{INTRODUÇÃO}

O Regulamento Técnico da Qualidade - RTQ para o Nível de Eficiência Energética de Edificações Residenciais (INMETRO, 2012) apresenta dois procedimentos para determinação do nível de eficiência energética da envoltória de edificações residenciais: um prescritivo e outro por simulação computacional. No caso de edificações naturalmente ventiladas, o método prescritivo consiste na aplicação de equações para o cálculo do somatório de graus-hora de resfriamento e consumo relativo para aquecimento. No caso de edificações condicionadas artificialmente considera-se também o consumo de energia para refrigeração.

As equações de cálculo do método prescritivo foram obtidas por análise de regressão multivariada. Por meio delas, indicadores de desempenho da edificação são relacionados com três grupos de variáveis que descrevem o projeto: térmicas (ex: absortância, capacidade térmica e transmitância térmica de componentes construtivos); geométricas (ex: área útil do ambiente, área de aberturas, pé-direito); e binárias (ex: se existe cobertura voltada para o exterior, se existe contato com o solo, se existe dispositivo de sombreamento nas janelas).

Porém, na lista de parâmetros considerados no método prescritivo, o tipo de vidro ou o fator solar de fechamentos envidraçados não é levado em consideração. Como o uso de vidros de controle solar pode trazer benefícios significativos para a melhoria do conforto térmico e redução de consumo de energia para climatização de edificações residenciais, o objetivo deste trabalho é propor novas equações de cálculo para o RTQ$\mathrm{R}$, incluindo o fator solar como o parâmetro que descreve a influência do vidro no nível de eficiência energética da envoltória da edificação.

Os experimentos foram realizados por meio de simulação computacional com o programa Energyplus e arquivos climáticos de dados horários de oito cidades brasileiras percorrendo todas as zonas bioclimáticas definidas na NBR 15220. Novas equações para descrever o desempenho das edificações residenciais foram obtidas por análise de regressão reproduzindo a mesma metodologia aplicada pelo CB3E (Centro Brasileiro de Eficiência Energética em Edificações), quando da obtenção das equações para desenvolvimento da versão atual do regulamento. Três diferentes especificações de vidro foram adicionadas ao lote de simulações adotado naquela metodologia, totalizando 34.560 casos simulados nesta proposta.

\section{FUNDAMENTAÇÃO TEÓRICA}

O desenvolvimento do método prescritivo para análise da envoltória adotado no RTQ-R é descrito por Sorgato (2011), Versage (2011) e Fossati (2011). Nas equações para análise do nível de eficiência da envoltória não há nenhum parâmetro que diferencie o tipo de vidro utilizado nas janelas das residências.

A Figura 1 mostra, como exemplo, a equação que deve ser aplicada a edificações construídas na Zona Bioclimática 1. Para as demais zonas bioclimáticas o mesmo formato de equação é utilizado, alterando-se alguns termos e os coeficientes das variáveis independentes. Os únicos fatores relacionados às janelas e que aparecem nas equações são: 
a) $A A b_{N}, A A b_{L}, A A b_{S}, A A b_{O}$ : áreas de abertura na orientação norte, leste, sul e oeste, respectivamente;

b) somb: variável que define a presença de dispositivos de proteção solar externos às aberturas, sendo adotado o valor zero quando não houver dispositivos de proteção solar e 1 quando houver;

c) vid: variável binária que indica a existência de vidro duplo no ambiente, sendo adotado valor 1 se houver vidro duplo e zero se não houver.

\section{Figura 1 - Equação de cálculo do indicador de graus-hora de resfriamento para a} Zona Bioclimática 1.

\begin{tabular}{|c|c|c|c|c|c|c|c|}
\hline \multicolumn{8}{|c|}{ 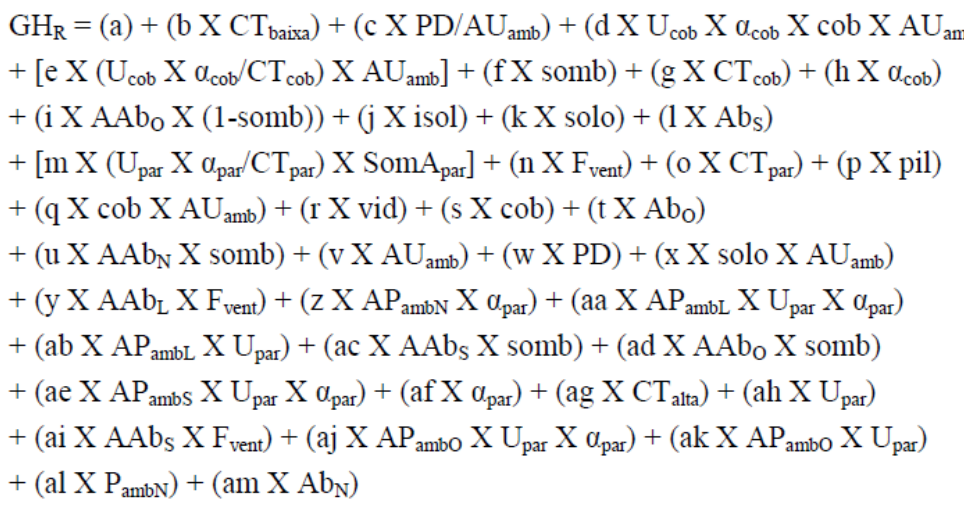 } \\
\hline $\mathrm{a}$ & 94,0522 & $\mathrm{k}$ & $-54,3782$ & $\mathrm{u}$ & 5,4197 & ae & 0,4732 \\
\hline $\mathrm{b}$ & 123,0188 & 1 & $-13,7824$ & $\mathrm{v}$ & $-0,8241$ & af & 15,3256 \\
\hline $\mathrm{c}$ & 164,3781 & $\mathrm{~m}$ & 0,2637 & $\mathrm{w}$ & 6,9951 & $\mathrm{ag}$ & 16,2345 \\
\hline d & 2,6353 & $\mathrm{n}$ & $-35,9380$ & $\mathrm{x}$ & 0,4141 & $\mathrm{ah}$ & $-12,2883$ \\
\hline $\mathrm{e}$ & 3,0564 & 0 & $-0,0441$ & $\mathrm{y}$ & 5,4487 & ai & 2,6390 \\
\hline $\mathrm{f}$ & $-66,6721$ & $\mathrm{p}$ & $-19,2971$ & $z$ & $-0,1569$ & aj & 1,7745 \\
\hline $\mathrm{g}$ & $-0,0607$ & $\mathrm{q}$ & $-0,9075$ & aa & 2,4596 & ak & $-0,7678$ \\
\hline $\mathrm{h}$ & 56,9221 & $\mathrm{r}$ & $-16,1623$ & $\mathrm{ab}$ & $-1,0187$ & $\mathrm{al}$ & 22,1077 \\
\hline $\mathrm{i}$ & 9,1358 & $\mathrm{~s}$ & 50,8387 & $\mathrm{ac}$ & 2,2785 & am & $-15,7841$ \\
\hline $\mathrm{J}$ & $-32,8413$ & $t$ & 21,8479 & $\mathrm{ad}$ & 2,9537 & & \\
\hline
\end{tabular}

Pelas equações do RTQ-R na versão atual, a utilização de um vidro de controle solar nas janelas da residência não afeta o índice de eficiência da envoltória. A mesma deficiência também foi detectada no método prescritivo para análise de eficiência energética da envoltória de edificações residenciais no RTQ-C, para edifícios comerciais, de serviços e públicos, conforme apresentado por Yamakawa e Westphal (2011) e Castro e Westphal (2011). Porém, no RTQ-C as equações do método prescritivo apresentam o fator solar como variável independente para cálculo do indicador do consumo de energia, embora os resultados não sejam afetados significativamente pelo tipo de vidro utilizado, mas sim, pela existência ou não de brises nas fachadas.

Melo (2012) também destaca a incapacidade das equações do método prescritivo do RTQ-C em representar a relação entre os dados de entrada e o consumo de energia da edificação, quando comparados com a avaliação por simulação computacional. As pesquisas acima destacam que o método prescritivo, tanto no RTQ-R, quanto no RTQ-C não conseguem representar a mesma precisão da simulação computacional integrada para análise da eficiência da envoltória.

O presente trabalho supõe que ao alterar o tipo de vidro nas janelas de uma edificação residencial pode-se obter diferentes padrões de conforto térmico interno, e 
consequentemente, interferir no consumo de energia para resfriamento e aquecimento do ar interno.

\section{METODOLOGIA}

Para propor um ajuste nas equações do método prescritivo do RTQ-R, a mesma metodologia aplicada por Sorgato (2011) foi aplicada, executando-se uma nova série de simulações paramétricas com a inclusão de três diferentes tipos de vidro. Novas equações para o cálculo de graus-hora de resfriamento, consumo relativo de aquecimento e refrigeração foram obtidas por análise de regressão multivariada.

O modelo computacional utilizado por Sorgato (2011) foi gerado para simulação no EnergyPlus, conforme ilustrado na Figura 2. O zoneamento térmico adotado no pavimento tipo do modelo, que compreende quatro apartamentos por andar, é ilustrado na Figura 3. A análise dos graus-hora de desconforto foi conduzida para as salas e dormitórios, indicados na figura.

Por questões de espaço, apenas as simulações realizadas para o pavimento tipo, e os cálculos dos graus-hora de desconforto para edificações naturalmente ventiladas serão apresentadas neste artigo. As simulações foram conduzidas para as oito zonas bioclimáticas brasileiras, sendo adotado o arquivo climático de uma cidade representativa de cada zona: Curitiba (ZB1), Santa Maria (ZB2), Florianópolis (ZB3), Brasília (ZB4), Macaé (ZB5), Campo Grande (ZB6), Cuiabá (ZB7) e Salvador (ZB8). Maiores detalhes sobre as considerações da metodologia de modelagem e simulação podem ser encontrados em Sorgato (2011).

$\mathrm{Na}$ lista de variáveis arquitetônicas e construtivas adotadas por Sorgato (2011) foram adicionados mais dois tipos de vidro, além do incolor de $4 \mathrm{~mm}$ : um vidro verde de $6 \mathrm{~mm}$, com Fator Solar igual a $60 \%$ e um vidro de controle solar, de cor cinza, com Fator Solar igual a $34 \%$. As propriedades térmicas e ópticas dos vidros utilizados nas simulações são apresentadas na Tabela 1.

Figura 2 - Modelo geométrico adotado nas simulações computacionais.

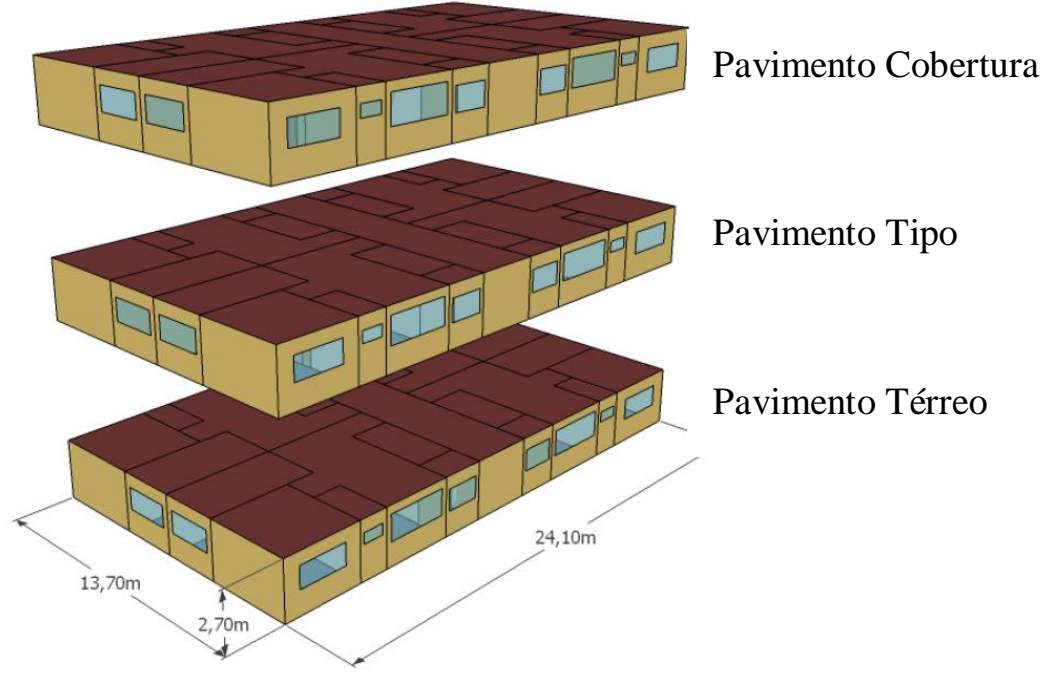


Figura 3 - Planta-baixa do pavimento tipo adotado nas simulações e indicação das zonas térmicas consideradas para análise dos graus-hora de desconforto.

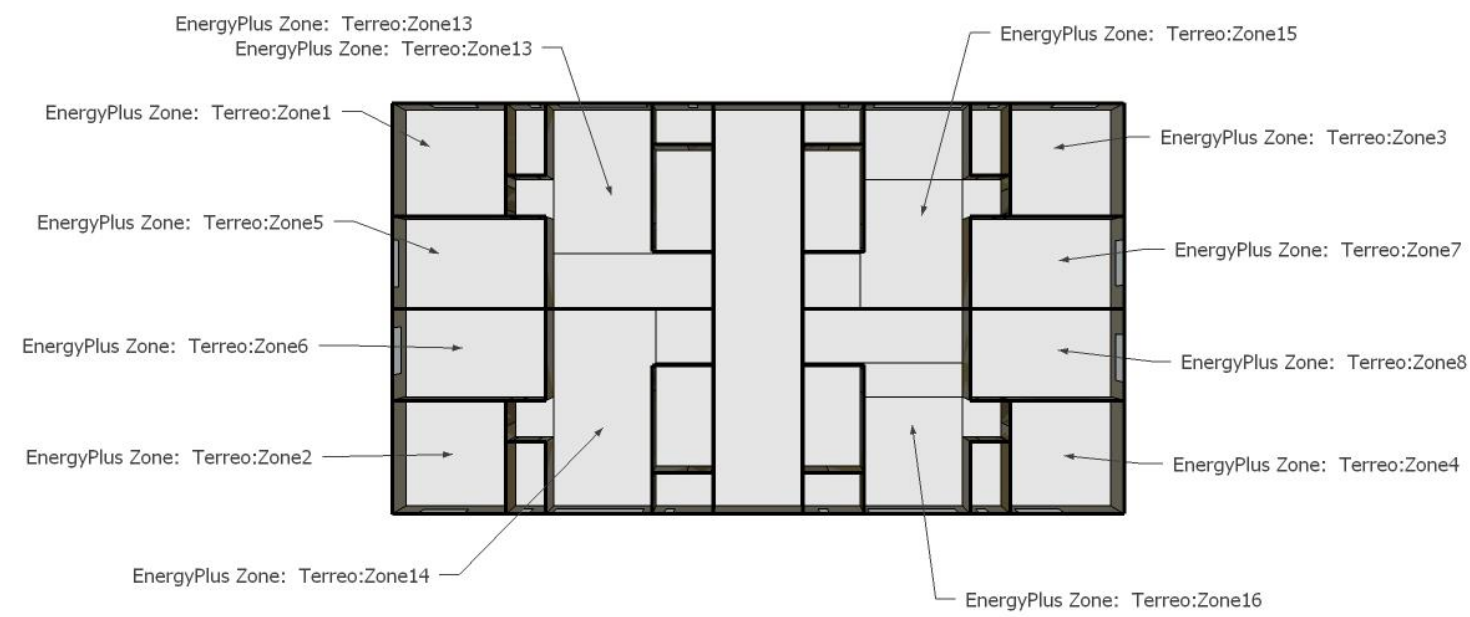

Tabela 1 - Propriedades ópticas e térmicas dos vidros adotados no estudo.

\begin{tabular}{|l|r|r|r|}
\hline Nome & Vidro 1 & Vidro 2 & Vidro 3 \\
\hline Fator Solar & $85 \%$ & $60 \%$ & $34 \%$ \\
\hline Espessura (m) & 0,004 & 0,005 & 0,008 \\
\hline Transmissão solar & 0,825 & 0,452 & 0,240 \\
\hline Reflexão solar da face externa & 0,074 & 0,054 & 0,370 \\
\hline Reflexão solar da face interna & 0,074 & 0,054 & 0,360 \\
\hline Transmissão visível & 0,896 & 0,756 & 0,510 \\
\hline Reflexão visível da face externa & 0,081 & 0,070 & 0,170 \\
\hline Reflexão visível da face interna & 0,081 & 0,070 & 0,180 \\
\hline Emissividade da face externa & 0,840 & 0,840 & 0,840 \\
\hline Emissividade da face interna & 0,840 & 0,840 & 0,840 \\
\hline Condutividade (W/m².K) & 1,00 & 1,00 & 1,00 \\
\hline
\end{tabular}

\section{RESULTADOS}

Este artigo apresenta os resultados parciais da proposta de ajuste para as equações de cálculo do somatório de graus-hora de resfriamento para as edificações naturalmente ventiladas. Ao todo, esse lote compreende 1.440 casos, que corresponde ao pavimento tipo de um modelo geométrico, dos três adotados por Sorgato (2011), e todas as oito zonas bioclimáticas. Como foram analisados 12 cômodos do modelo, a base de dados de graus-hora contempla 17.280 linhas de resultados.

O Quadro 1 apresenta a nova equação proposta para a Zona Bioclimática 1 (ZB1) e seus respectivos coeficientes de ajuste das variáveis independentes. Neste estudo, o mesmo 
formato de equação foi aplicado para as oito zonas bioclimáticas. Entretanto, para alguns climas, determinados parâmetros não são significativos e neste caso o coeficiente é nulo.

A equação para a ZB1 foi a que apresentou coeficiente de determinação mais baixo, $\mathrm{R}^{2}$ igual a 0,66 . Esse valor ainda é superior ao modelo de equação atual do RTQ-R, com $\mathrm{R}^{2}$ igual a 0,42 , mas a proposta de ajuste ainda não contempla os três modelos geométricos adotados na elaboração do método prescritivo do RTQ-R. Provavelmente, na versão final da equação de ajuste o coeficiente de determinação esteja muito próximo do alcançado nas equações atuais do RTQ-R. Mas esse resultado indica que é possível adicionar o Fator Solar como mais um parâmetro de avaliação do desempenho das edificações residenciais, com o mesmo padrão de eficácia do método prescritivo atual.

\section{Quadro 1 - Equação proposta para a Zona Bioclimática 1.}

\begin{tabular}{|c|c|c|c|c|c|c|c|}
\hline \multicolumn{8}{|c|}{ 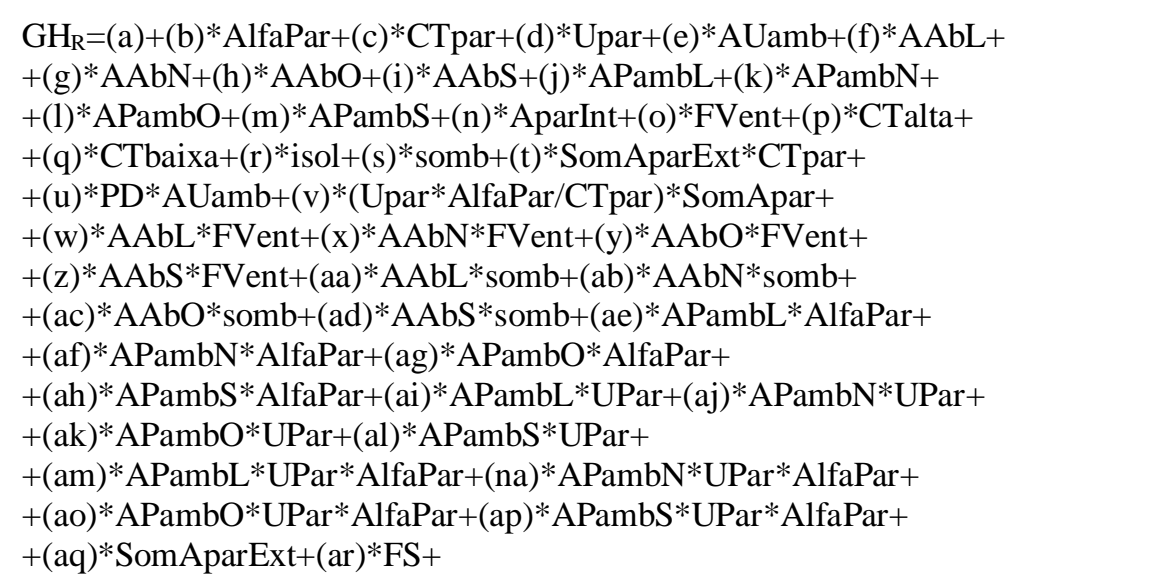 } \\
\hline $\mathrm{a}$ & 989,9 & 1 & 52,61 & W & $-3,812$ & ah & 60,22 \\
\hline b & $-358,1$ & $\mathrm{~m}$ & $-24,57$ & $\mathrm{x}$ & 21,22 & ai & $-30,77$ \\
\hline $\mathrm{c}$ & $-0,3733$ & $\mathrm{n}$ & 0 & $\mathrm{y}$ & $-9,302$ & aj & 3,849 \\
\hline d & $-209,3$ & o & $-241,2$ & $\mathrm{z}$ & 35,64 & ak & $-34,32$ \\
\hline e & 2,891 & $\mathrm{p}$ & 264,3 & aa & 17,46 & al & 7,107 \\
\hline $\mathrm{f}$ & $-23,57$ & $\mathrm{q}$ & $-88,27$ & $a b$ & 51,07 & am & 46,14 \\
\hline g & $-81,75$ & $\mathrm{r}$ & 214,3 & $\mathrm{ac}$ & 4,993 & na & $-11,97$ \\
\hline $\mathrm{h}$ & $-12,91$ & $\mathrm{~s}$ & $-367,2$ & $\mathrm{ad}$ & 83,1 & ao & 55,55 \\
\hline $\mathrm{i}$ & $-112,5$ & $\mathrm{t}$ & $-0,00436$ & ae & $-64,62$ & ap & $-19,8$ \\
\hline $\mathrm{j}$ & 47,99 & $\mathrm{u}$ & 0 & af & 65,79 & $\mathrm{aq}$ & 0 \\
\hline $\mathrm{k}$ & $-22,57$ & $\mathrm{v}$ & 5,178 & $\mathrm{ag}$ & $-72,01$ & ar & 212,1 \\
\hline
\end{tabular}

A Figura 4 mostra a relação entre o somatório de graus-hora de desconforto para resfriamento calculados pela equação proposta para a ZB1 e somatório de graus-hora obtidos por simulação no gráfico à esquerda. Nota-se a baixa aderência dos resultados, com $\mathrm{R}^{2}$ de 0,6641 , ou seja, muito aquém de uma correlação ideal, que seria $\mathrm{R}^{2}$ igual a 1.

Novas faixas de classificação do nível de eficiência da envoltória foram geradas seguindo a mesma metodologia apresentada por Versage (2011). Os modelos simulados foram classificados de acordo com essa metodologia. A frequência de ocorrência de modelos por faixa de classificação é apresentada no gráfico à direita, na Figura 4. 
Observa-se que há uma grande concentração de casos com nível A. Como essa cidade apresenta baixo somatório de graus hora de desconforto por calor, grande parte dos casos acaba alcançando elevado nível de eficiência. Isso indica que a metodologia de definição dos intervalos de $\mathrm{GH}_{\mathrm{R}}$ que estabelecem os níveis de eficiência também não está adequada e deverá ser refeita de acordo com os resultados das novas equações.

Figura 4 - Relação entre graus-hora de desconforto calculado e simulado (esq.), e distribuição dos casos por faixa de classificação (dir.) para a zona bioclimática 1.
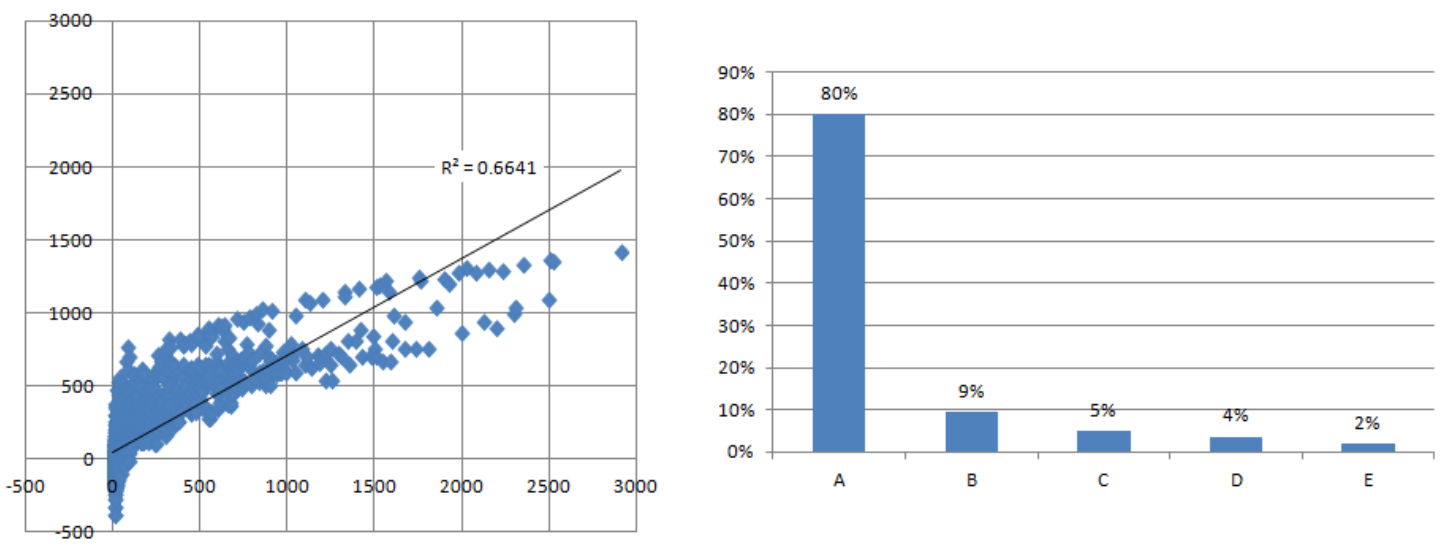

A equação para a ZB7 foi a que apresentou o melhor coeficiente de determinação, com $\mathrm{R}^{2}$ igual a 0,94 . Adotando-se o mesmo formato de equação que foi gerado para a ZB1, os coeficientes para a ZB7 são listados no Quadro 2.

Quadro 2 - Coeficientes da equação proposta para a Zona Bioclimática 7.

\begin{tabular}{|l|r|}
\hline $\mathrm{a}$ & 29830 \\
\hline $\mathrm{b}$ & 5729 \\
\hline $\mathrm{c}$ & 0,03763 \\
\hline $\mathrm{d}$ & -4016 \\
\hline $\mathrm{e}$ & 260,9 \\
\hline $\mathrm{f}$ & $-448,9$ \\
\hline $\mathrm{g}$ & $-866,2$ \\
\hline $\mathrm{h}$ & $-411,5$ \\
\hline $\mathrm{i}$ & -1449 \\
\hline $\mathrm{j}$ & -1093 \\
\hline $\mathrm{k}$ & -1417 \\
\hline
\end{tabular}

\begin{tabular}{|l|r|}
\hline$l$ & -1185 \\
\hline $\mathrm{m}$ & -1380 \\
\hline $\mathrm{n}$ & 0 \\
\hline $\mathrm{o}$ & -8787 \\
\hline $\mathrm{p}$ & -1871 \\
\hline $\mathrm{q}$ & 3541 \\
\hline $\mathrm{r}$ & -6216 \\
\hline $\mathrm{s}$ & -8396 \\
\hline $\mathrm{t}$ & 0,000936 \\
\hline $\mathrm{u}$ & 0 \\
\hline $\mathrm{v}$ & $-4,079$ \\
\hline
\end{tabular}

\begin{tabular}{|l|r|}
\hline $\mathrm{W}$ & -1085 \\
\hline $\mathrm{x}$ & -1111 \\
\hline $\mathrm{y}$ & -1090 \\
\hline $\mathrm{z}$ & -1019 \\
\hline $\mathrm{aa}$ & $-292,5$ \\
\hline ab & $-166,3$ \\
\hline ac & $-426,7$ \\
\hline ad & 471,5 \\
\hline ae & 1509 \\
\hline af & 2250 \\
\hline ag & 1567 \\
\hline
\end{tabular}

\begin{tabular}{|l|r|}
\hline ah & 2008 \\
\hline ai & 496,9 \\
\hline aj & 680,3 \\
\hline ak & 523,3 \\
\hline al & 648,6 \\
\hline am & $-416,1$ \\
\hline na & $-807,2$ \\
\hline ao & $-440,9$ \\
\hline ap & $-800,1$ \\
\hline aq & 0 \\
\hline ar & 5721 \\
\hline
\end{tabular}

A Figura 5 mostra a relação entre $\mathrm{GH}_{\mathrm{R}}$ calculado e simulado para os casos da ZB7 no gráfico à esquerda. Nota-se uma aderência muito maior entre os dados, com $\mathrm{R}^{2}$ de 0,9433 para o modelo matemático proposto, do que o obtido para a ZB1. Esse melhor comportamento pode ser explicado pelo fato da ZB7 ser constantemente mais quente e ter uma uniformidade maior de casos com grande quantidade de horas de desconforto por calor. O gráfico da direita mostra a distribuição de casos por nível de eficiência de acordo com a nova equação e faixas de classificação. Observa-se uma melhor distribuição do que a obtida na ZB1, com maior concentração no nível de eficiência B. 
Figura 5 - Relação entre graus-hora de desconforto calculado e simulado (esq.), e distribuição dos casos por faixa de classificação (dir.) para a zona bioclimática 7 .
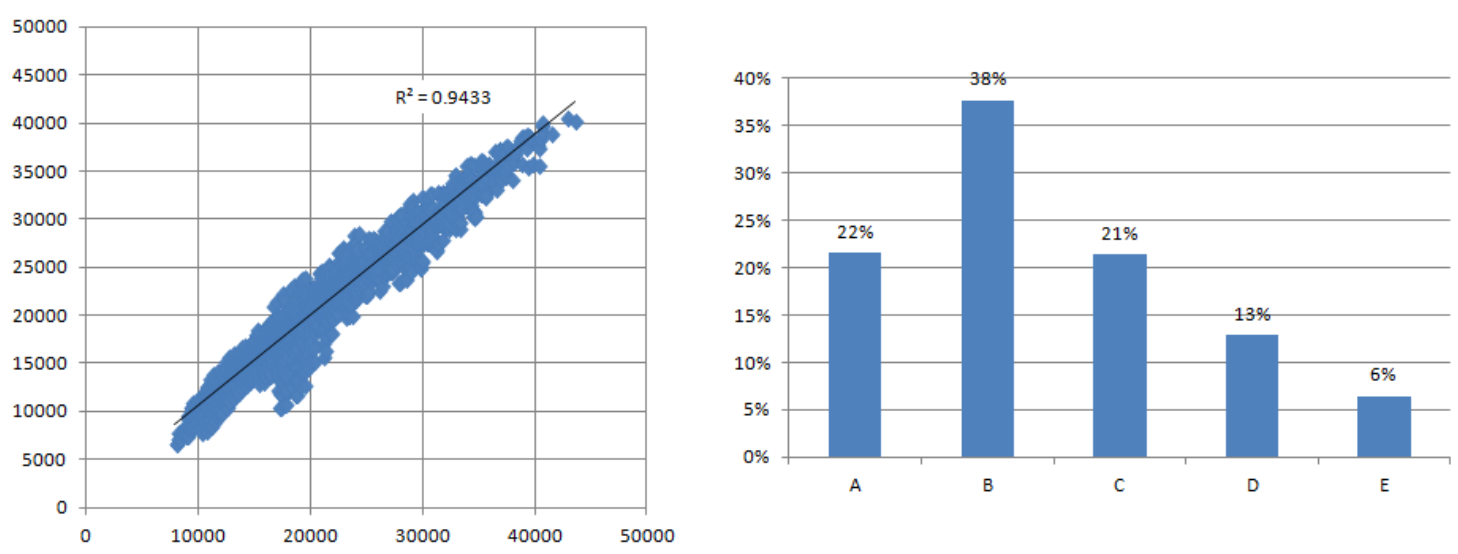

Para verificar a sensibilidade do novo modelo de equação ao tipo de vidro adotado nas edificações, três testes foram desenvolvidos avaliando-se o nível de eficiência de um quarto com 9,0 $\mathrm{m}^{2}$ de parede externa, $18,3 \mathrm{~m}^{2}$ de paredes internas e 2,0 $\mathrm{m}^{2}$ de área de abertura. As paredes foram mantidas com transmitância térmica igual a $2,30 \mathrm{~W} / \mathrm{m}^{2} \cdot \mathrm{K}$ e variou-se a orientação da parede externa e sua abertura, assim como o fator de ventilação da abertura. O Quadro 3 apresenta os níveis de eficiência obtidos nas oito zonas bioclimáticas para o caso com parede externa voltada para leste e fator de ventilação de $50 \%$ da abertura. São apresentados os níveis de eficiência para três tipos de vidro, com fator solar de $85 \%, 60 \%$ e $34 \%$. Observa-se que nessas condições a alteração do tipo de vidro ocasionou mudança do nível de eficiência em quatro zonas bioclimáticas (ZB1, ZB4, ZB5 e ZB7).

Quadro 3 - Caso com parede externa voltada para leste e fator de ventilação $50 \%$.

\begin{tabular}{|l|r|c|r|c|r|c|}
\hline \multirow{2}{*}{$\begin{array}{l}\text { Zona } \\
\text { bioclimática }\end{array}$} & \multicolumn{2}{|c|}{ FS $=\mathbf{8 5 \%}$} & \multicolumn{2}{c|}{ FS $=\mathbf{6 0 \%}$} & \multicolumn{2}{c|}{ FS $=\mathbf{3 4 \%}$} \\
\cline { 2 - 8 } & GHR & Nível & GHR & Nível & GHR & Nível \\
\hline ZB1 & 516 & D & 463 & D & 427 & C \\
\hline ZB2 & 3400 & B & 3056 & B & 2821 & B \\
\hline ZB3 & 3192 & D & 2910 & D & 2718 & D \\
\hline ZB4 & 2545 & D & 2256 & D & 2059 & C \\
\hline ZB5 & 5625 & B & 5165 & A & 4852 & A \\
\hline ZB6 & 7842 & C & 6973 & C & 6382 & C \\
\hline ZB7 & 24906 & D & 23476 & C & 22503 & C \\
\hline ZB8 & 18593 & E & 17592 & E & 16912 & E \\
\hline
\end{tabular}

No segundo teste a parede externa foi orientada a norte e o fator de ventilação foi ampliado para $100 \%$. Nesse caso, buscou-se diminuir o impacto do tipo de vidro nas condições de conforto interno. Mesmo assim, a variação do tipo de vidro foi determinante para mudar o nível de eficiência em cinco zonas bioclimáticas (ZB3, ZB4, ZB6, ZB7, ZB8).

No terceiro e último teste, com parede orientada a norte e fator de ventilação $50 \%$, a mudança do vidro gerou alteração do nível de eficiência em três zonas bioclimáticas (ZB4, ZB5 e ZB7). 
Os três testes indicam que o tipo de vidro, representado neste estudo pelo fator solar, pode influenciar no nível de conforto avaliado por graus-hora de resfriamento de acordo com a metodologia de análise do RTQ-R, desde que as equações sejam ajustadas. Em sete das oito zonas bioclimáticas houve variação do nível de eficiência do ambiente analisado quando se reduziu o fator solar pela metade. Apenas a ZB2 não foi sensível a essa alteração. Porém uma nova metodologia de definição das faixas de eficiência pode ser desenvolvida a partir dos resultados obtidos com as novas equações.

Quadro 4 - Parede externa voltada para o norte e fator de ventilação $100 \%$.

\begin{tabular}{|l|r|c|r|c|r|c|}
\hline \multirow{2}{*}{$\begin{array}{l}\text { Zona } \\
\text { bioclimática }\end{array}$} & \multicolumn{2}{|c|}{ FS $=\mathbf{8 5 \%}$} & \multicolumn{2}{|c|}{ FS $=\mathbf{6 0 \%}$} & \multicolumn{2}{c|}{ FS $=\mathbf{3 4 \%}$} \\
\cline { 2 - 7 } & GHR & Nível & GHR & Nível & GHR & Nível \\
\hline ZB1 & 395 & C & 342 & C & 306 & C \\
\hline ZB2 & 2288 & A & 1944 & A & 1710 & A \\
\hline ZB3 & 1878 & C & 1596 & B & 1404 & B \\
\hline ZB4 & 1734 & C & 1445 & B & 1248 & B \\
\hline ZB5 & 3516 & A & 3056 & A & 2743 & A \\
\hline ZB6 & 5725 & C & 4856 & B & 4265 & B \\
\hline ZB7 & 18851 & C & 17420 & B & 16448 & B \\
\hline ZB8 & 14723 & E & 13723 & D & 13043 & D \\
\hline
\end{tabular}

Quadro 5 - Parede externa voltada para o norte e fator de ventilação $50 \%$.

\begin{tabular}{|l|r|c|r|r|r|c|}
\hline \multirow{2}{*}{$\begin{array}{l}\text { Zona } \\
\text { bioclimática }\end{array}$} & \multicolumn{2}{|c|}{ FS $=\mathbf{8 5 \%}$} & \multicolumn{2}{c|}{ FS $=\mathbf{6 0 \%}$} & \multicolumn{2}{|c|}{ FS $=\mathbf{3 4 \%}$} \\
\cline { 2 - 8 } & GHR & Nível & GHR & Nível & GHR & Nível \\
\hline ZB1 & 551 & D & 498 & D & 462 & D \\
\hline ZB2 & 3660 & B & 3315 & B & 3081 & B \\
\hline ZB3 & 3119 & D & 2837 & D & 2645 & D \\
\hline ZB4 & 2659 & D & 2370 & D & 2173 & C \\
\hline ZB5 & 5510 & B & 5050 & A & 4737 & A \\
\hline ZB6 & 8008 & C & 7139 & C & 6548 & C \\
\hline ZB7 & 24698 & D & 23267 & C & 22295 & C \\
\hline ZB8 & 21011 & E & 20011 & E & 19331 & E \\
\hline
\end{tabular}

\section{CONSIDERAÇÕES FINAIS}

Este artigo apresentou uma proposta de ajuste nas equações de cálculo de graus-hora de desconforto por calor utilizadas no método prescritivo do RTQ-R para classificação do nível de eficiência energética de edificações residenciais. $\mathrm{O}$ ajuste consiste na inclusão do fator solar dos vidros como mais um parâmetro de influência do desempenho da edificação. Novas equações foram obtidas por análise de regressão multivariada com coeficientes de determinação próximos aos obtidos pelos modelos adotados no RTQ-R. Testes de avaliação do nível de eficiência energética da envoltória de um cômodo de uma edificação foram realizados e identificou-se variação do nível de eficiência em sete das oito zonas bioclimáticas brasileiras. Os testes consideraram a redução do fator solar do vidro de $85 \%$ para $60 \%$ e $34 \%$, simulando situações onde um vidro incolor seria 
substituído por um vidro verde e um vidro de controle solar, de cor cinza. Nesses testes, a redução no somatório de graus-hora de resfriamento foi de $8 \%$ a $28 \%$, quando o fator solar foi reduzido de $85 \%$ para $34 \%$, revelando o impacto significativo do vidro no conforto térmico dos dormitórios e salas.

Os resultados desse trabalho podem contribuir para melhoria do método prescritivo do RTQ-R, de forma que represente com melhor precisão a influência de parâmetros da envoltória na eficiência energética de edificações.

Porém, o autor acredita que as equações adotadas no RTQ-R não são a melhor ferramenta para prescrever condições de envoltória para a classificação do nível de eficiência energética de edificações residenciais. A quantidade de parâmetros adotados nas equações não torna o modelo de análise simples e educativa como um método prescritivo como deveria ser. Talvez a especificação de limites de valores para transmitância térmica, capacidade térmica e fator solar fosse a metodologia mais adequada para prescrever condições limites de eficiência da envoltória. A forma complexa das equações do RTQ-R indica que a metodologia adotada para avaliação de desempenho está reescrevendo o modelo de simulação computacional na forma de uma equação só. Ao aproximar-se do nível de complexidade de uma simulação detalhada, a função de um método prescritivo desse tipo é altamente questionável e até dispensável. Além disso é importante ressaltar que no cálculo do balanço térmico o EnergyPlus considera sempre as janelas fechadas, mesmo que o módulo de ventilação indique o contrário. Isso pode gerar diferenças significativas no cálculo da carga térmica interna, principalmente quando utilizado um vidro de controle solar.

\section{REFERÊNCIAS}

CASTRO, L. T.; WESTPHAL, F. S. Etiqueta Nacional de Conservação de Energia: comparativo entre o método prescritivo e simulação para análise de desempenho da envoltória. Anais... In: XI Encontro Nac. de Conforto no Ambiente Construído, 2011, Búzios, RJ, 2011

FOSSATI, M. Relatório técnico final de elaboração do texto RTQ-R. Universidade Federal de Santa Catarina, Departamento de Engenharia Civil. Florianópolis, 2011. 25p. Disponível em: <http://www.labeee.ufsc.br/projetos/etiquetagem/desenvolvimento/atividades-2008-2011/ trabalho-ii> Acesso em: 24 abr. 2014.

INMETRO. Portaria n. 18, de 16 de janeiro de 2012. Disponível em: < http://www.inmetro.gov.br/legislacao/rtac/pdf/RTAC001788.pdf >. Acesso em: 24 abr. 2014.

MELO, A. P. Desenvolvimento de um método para estimar o consumo de energia de edificações comerciais através da aplicação de redes neurais. Tese de doutorado. Universidade Federal de Santa Catarina. PPGEC. Florianópolis, 2012. 189 p.

SORGATO, M. J. Relatório técnico da base de simulações para o RTQ-R. Universidade Federal de Santa Catarina, Departamento de Engenharia Civil. Florianópolis, 2011. 55p. Disponível em: <http://www.labeee.ufsc.br/projetos/etiquetagem/desenvolvimento/atividades2008-2011/trabalho-ii> Acesso em: 24 abr. 2014.

VERSAGE, R. S. Equações prescritivas para o regulamento de etiquetagem de eficiência energética de edificações residenciais. Universidade Federal de Santa Catarina, Departamento de Engenharia Civil. Florianópolis, 2011. 72p. Disponível em: <http://www.labeee.ufsc.br/ projetos/etiquetagem/desenvolvimento/atividades-2008-2011/trabalho-ii>Acesso: 24 abr. 2014.

YAMAKAWA, M. A.; WESTPHAL, F. S. Influência do percentual de abertura nas fachadas e do fator solar dos vidros na etiquetagem do PROCEL/INMETRO: método prescritivo $\mathrm{X}$ simulação. Anais... In: XI Encontro Nacional de Conforto no Ambiente Construído, 2011, Búzios, RJ, 2011. 